\title{
Sustained good glycaemic control in NIDDM patients by implementation of structured care in general practice: 2-year follow-up study
}

\author{
J.J.J. de Sonnaville ${ }^{1,5}$, M. Bouma ${ }^{2}$, L.P. Colly ${ }^{3}$, W.Devillé ${ }^{4}$, D. Wijkel ${ }^{1}$, R.J.Heine ${ }^{5}$ \\ ${ }^{1}$ Research Centre Primary/Secondary Health Care, Academic Hospital, Vrije Universiteit, Amsterdam, The Netherlands \\ ${ }^{2}$ Department of General Practice, Social Medicine and Nursing Home Medicine, Vrije Universiteit, Amsterdam, The Netherlands \\ ${ }^{3}$ Amsterdam Thrombosis Service and Laboratory for General Practitioners, Amsterdam, The Netherlands \\ ${ }^{4}$ Department of Epidemiology and Biostatistics, Vrije Universiteit, Amsterdam, The Netherlands \\ ${ }^{5}$ Institute for Endocrinology, Reproduction and Metabolism, Vrije Universiteit, Amsterdam, The Netherlands
}

Summary In primary care it is difficult to treat the growing number of non-insulin-dependent diabetic (NIDDM) patients according to (inter)national guidelines. A prospective, controlled cohort study was designed to assess the intermediate term (2 years) effect of structured NIDDM care in general practice with and without 'diabetes service' support on glycaemic control, cardiovascular risk factors, general well-being and treatment satisfaction. The 'diabetes service', supervised by a diabetologist, included a patient registration system, consultation facilities of a dietitian and diabetes nurse educator, and protocolized blood glucose lowering therapy advice which included home blood glucose monitoring and insulin therapy. In the study group (SG; 22 general practices), 350 known NIDDM patients over 40 years of age (206 women; mean age 65.3 \pm SD 11.9; diabetes duration $5.9 \pm 5.4$ years) were followed for 2 years. The control group (CG; 6 general practices) consisted of 68 patients ( 28 women; age $64.6 \pm 10.3$; diabetes duration $6.3 \pm 6.4$ years). Mean $\mathrm{HbA}_{1 \mathrm{c}}$ (reference 4.3-6.1\%) fell from 7.4 to $7.0 \%$ in SG and rose from 7.4 to $7.6 \%$ in CG during follow-up $(p=0.004)$. The percentage of patients with poor control $\left(\mathrm{HbA}_{1 \mathrm{c}}>8.5 \%\right)$ shifted from 21.4 to $11.7 \%$ in $\mathrm{SG}$, but from 23.5 to $27.9 \%$ in CG $(p=0.008)$. Good control $\left(\mathrm{HbA}_{1 \mathrm{c}}<7.0 \%\right)$ was achieved in $54.3 \%$ (SG; at entry $43.4 \%$ ) and $44.1 \%$ (CG; at entry $54.4 \%$ ) $(p=0.013)$. Insulin therapy was started in $29.7 \%$ (SG) and $8.8 \%(\mathrm{CG})$ of the patients $(p=0.000)$ with low risk of severe hypoglycaemia (0.019/patient year). Mean levels of total and HDL-cholesterol (SG), triglycerides (SG) and diastolic blood pressure $(\mathrm{SG}+\mathrm{CG})$ and the percentage of smokers (SG) declined significantly, but the prevalence of these risk factors remained high. General well-being (SG) did not change during intensified therapy. Treatment satisfaction (SG) tended to improve. Implementation of structured care, including education and therapeutic advice, results in sustained good glycaemic control in the majority of NIDDM patients in primary care, with low risk of hypoglycaemia. Lowering cardiovascular risk requires more than reporting results and referral to guidelines. [Diabetologia (1997) 40: 13341340]

Keywords Structured diabetes care, NIDDM, primary care, diabetes education, insulin therapy, hypoglycaemia, well-being.
Received: 5 February 1997 and in revised form: 22 May 1997

Corresponding author: J.J.J. de Sonnaville, Department of Endocrinology, Academic Hospital Vrije Universiteit, P. O. Box 7057, NL-1007 MB Amsterdam, The Netherlands

Abbreviations: GP, General practitioner; NIDDM, non-insulin-dependent diabetes mellitus; HBGM, home blood glucose monitor.
Most patients with non-insulin-dependent diabetes mellitus (NIDDM) in particular when managed with diet or oral hypoglycaemic agents, are treated in a primary health care setting. To optimize the treatment of the growing number of NIDDM patients (inter-) national guidelines have been published [1-4]. However, the wide acceptance of the guidelines by general practitioners (GPs), and their implementation in practice have proved difficult [5-11]. 
Table 1. NIDDM patients above 40 years of age in study and control groups

\begin{tabular}{|c|c|c|c|c|}
\hline & \multicolumn{3}{|c|}{$\begin{array}{l}\text { Study group (SG) } \\
22 \text { GPs }\end{array}$} & \multirow{2}{*}{$\begin{array}{l}\text { Control group (CG) } \\
6 \text { GPs } \\
171 \\
\text { KDM }\end{array}$} \\
\hline Eligible NIDDM patients in GP records ${ }^{\mathrm{a}}(n)$ & & & $\begin{array}{l}570 \\
\text { KDM }\end{array}$ & \\
\hline 2 year (SG) and 1.5 year (CG) follow-up data available & $\begin{array}{l}95 \\
\text { NDM }\end{array}$ & & $\begin{array}{l}350 \\
\text { KDM }\end{array}$ & $\begin{array}{l}68 \\
\text { KDM }\end{array}$ \\
\hline
\end{tabular}

${ }^{a}$ Mean number of 1800 patients per GP; 369 (39\%) of all 939 identified NIDDM patients (SG) were managed in hospital diabetes clinics. ${ }^{\mathrm{b}}$ Patients with known diabetes for $>3$ months

(KDM) referred back from secondary care or referred by other GPs. NDM, Newly diagnosed NIDDM

Non-attendance and poor patient compliance are often major barriers for attaining adequate diabetes care. However, doctor-related, as for example attitude and knowledge, and organisational factors may be even more important barriers [12-14]. The availability of a diabetic patient register and recall system, education facilities, professional support from a network of medical and non-medical professionals and systematic quality assessment are organisational factors which influence diabetes care [15]. The guidelines for the treatment of NIDDM have stimulated physicians to improve the quality of management. However, implementation was only partially successful, where doctor-related and organisational problems could roughly be met $[9,16-20]$.

In 1992 we introduced a 'diabetes service' in primary care which provides support to GPs in the management of NIDDM. This facility is located at a laboratory for GPs in Amsterdam. The purpose of this study was to assess the intermediate term ( 2 years) effect of this care system on glycaemic control, various cardiovascular risk factors and on general well-being and treatment satisfaction in a population of NIDDM patients.

\section{Subjects, materials and methods}

Study population. Of 29 eligible GPs in the western part of Amsterdam 22 were willing to co-operate with the diabetes service. Reasons for non-participation were implementation of systemised diabetes care in their own practice (four GPs) and 'no interest' (three GPs). The 22 GPs were requested to enroll all their known and newly diagnosed NIDDM patients (Table 1). Only patients with diabetes (World Health Organisation criteria) diagnosed after the age of 40 years and at least treated for 6 months with diet and/or oral hypoglycaemic tablets were included. Of the 570 eligible patients managed in primary care 167 (29\%) did not participate. Reasons for non-participation were: deceased $(5.6 \%)$; referred to secondary care $(6.8 \%)$; change of GP $(7.2 \%)$; diabetes not confirmed $(2.2 \%)$; unwilling because of psychiatric disorders or unknown reasons $(7.3 \%)$. Two-year follow-up data are available for 350 of 459 known $(76.3 \%$ ) and 95 of 120 newly diagnosed $(79.2 \%)$ diabetic subjects. Reasons for $23.1 \%$ drop-out during the first 2 years of follow-up were death $(7.6 \%)$, change of GP
(7.9\%), referral to secondary care $(2.4 \%)$, delayed yearly control $(1.7 \%)$ and unknown reasons $(3.5 \%)$.

Control population. The NIDDM population of three GPs in Amsterdam and three in Twente (eastern part of the Netherlands) served as a control group. These GPs were not involved in any shared care system, but had accepted the treatment guidelines of the Dutch College of General Practitioners [4, 5]. Complete baseline and 1.5 year follow-up data for 68 of 102 participants are available (Table 2). Reasons for the $33.3 \%$ drop-out during 1.5 years of follow-up included death $(2.9 \%)$, change of GP $(3.9 \%)$, referral to secondary care $(1.0 \%)$, non-attendance or unknown reasons $(25.5 \%)$.

Diabetes service system Amsterdam. In this system the GP is supported by a laboratory with facilities to visit patients at home, a computerized patient register and recall system, a wide-angle retinal camera and the possibility to consult with a dietitian, a diabetes nurse educator and a podiatrist [21-23]. A diabetologist, who supervises the diabetes service, can be contacted by telephone $24 \mathrm{~h}$ a day.

Protocol. At entry, patients of the study population were invited for assessment of glycaemic control at 3-month intervals, and for annual review of complications, cardiovascular risk factors and assessment of general well-being and treatment satisfaction. Within 2 weeks the participating GPs received the results accompanied by an advice according to a step-up treatment regimen (Table 3 ). Target values were based upon the guidelines of the European NIDDM Policy Group 1988, which are similar to the Dutch GP Guidelines 1989 [2, 4]. GPs could alter target values, e.g. in the case of short life expectancy. If the target $\mathrm{HbA}_{1 \mathrm{c}}$-value (usually $7.0 \%$ ) was not met with maximal dose oral therapy, self-monitoring of blood glucose (first step) and insulin therapy (next step) were instructed in individual $30 \mathrm{~min}$ sessions with the diabetes educator. Usually, two follow-up appointments were sufficient to repeat and check education. Twice weekly telephone appointments were made to adjust insulin dose on the basis of fasting blood glucose values. Measurement of fasting and pre-dinner glucose values was requested for patients on a twice daily NPH-insulin scheme. Patients were free to choose one of the blood glucose meters and insulin pens available in the Netherlands. For the management of hypertension and dyslipoproteinaemia GPs were referred to GP guidelines [4]. The incidence of hypoglycaemia was evaluated annually, but in insulin-treated patients at least 3-monthly, at each visit or telephone call with the diabetes nurse. Patients were asked to verify hypoglycaemia by home blood glucose monitor (HBGM) if possible. Patients in the control group were seen at 3-month intervals for glycaemic control and annually for review of diabetic complications and 
Table 2. Baseline clinical characteristics of NIDDM patients in study and control groups

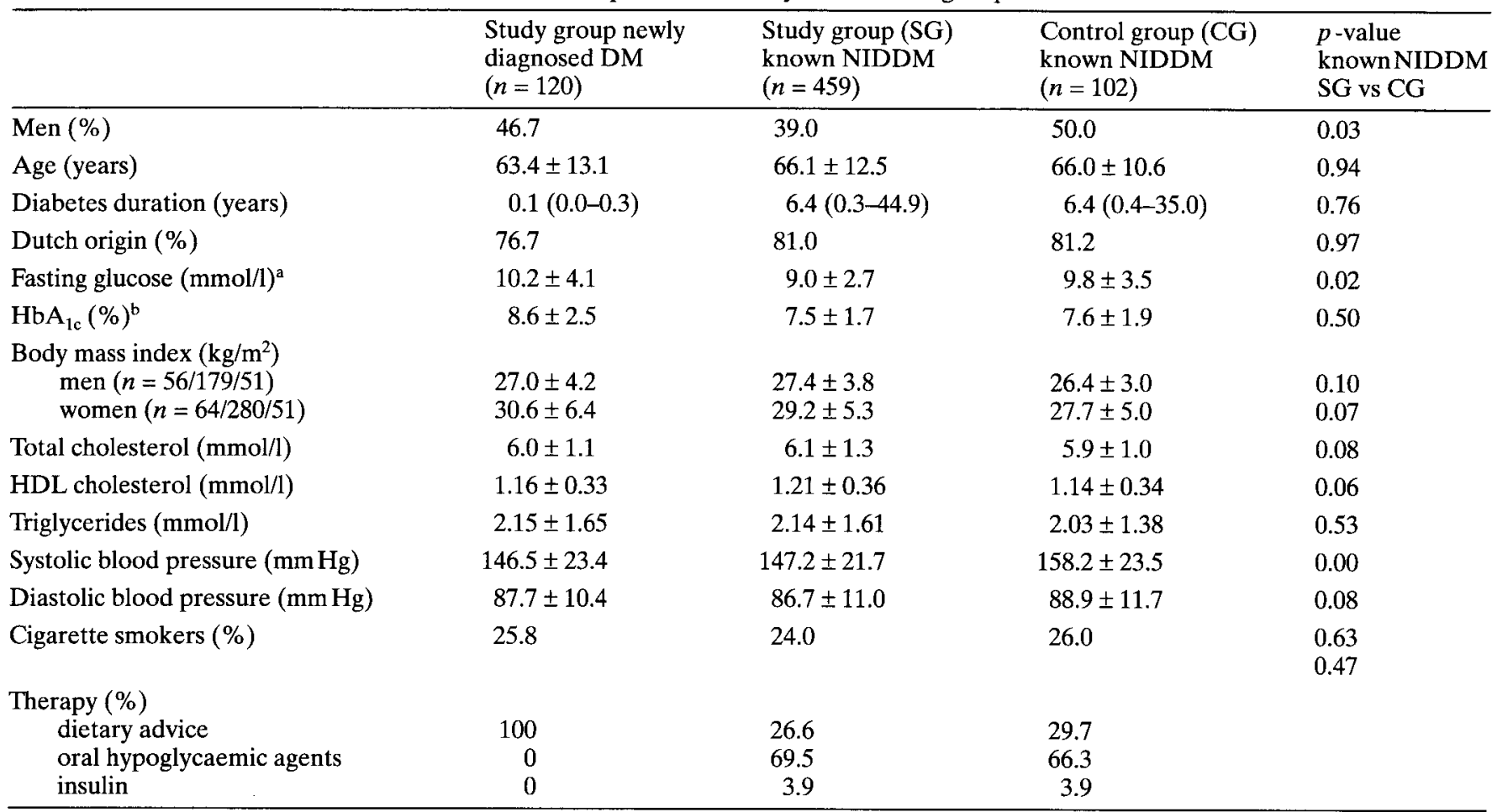

Values are expressed as mean \pm standard deviation or (range). ${ }^{a}$ Fasting glucose measured in venous plasma. ${ }^{b}$ Reference value $\mathrm{HbA}_{1 \mathrm{c}} 4.3-6.1 \%$

Table 3. Step-up regimen for glycaemic treatment of NIDDM patients in study group

1. Nutritional advice
Physical activity

2. Sulphonylureas (SU) Start at low dose; dose adjustments at 6-week intervals based upon fasting glucose and/or HbA

3. $\mathrm{SU}+$ metformin

4. Self-monitoring of blood glucose

5. NPH-insulin ${ }^{b}$ at bed-time + SU daytime

6. NPH-insulin twice daily In obese patients (BMI $>27 \mathrm{~kg} / \mathrm{m}^{2}$ ) metformin $500 \mathrm{mg}$ is added, increasing to a maximal dose of $1700 \mathrm{mg} /$ day Started in patients on maximal oral therapy if good glycaemic control is not achieved

The starting dose of 8 IU NPH-insulin is adjusted twice weekly, till fasting glucose targets are achieved

SU replaced by starting dose of 8 IU NPH-insulin before breakfast when daytime glucose levels $>10 \mathrm{mmol} /$ 1 with $\mathrm{HbA}_{1 \mathrm{c}}>$ target value. Morning + pre-dinner insulin doses titrated upon pre-dinner and fasting blood glucose levels

7. Add short-acting insulin If glycaemic control not satisfactory other insulin regimens may be appropriate or referral to diabetologist

In symptomatic patients who present with fasting glucose values $>15 \mathrm{mmol} / \mathrm{l}$ insulin is treatment of choice. a In general, glycaemic targets defined as fasting and preprandial blood glu-

cardiovascular risk factors. Therapy was titrated on the basis of fasting blood glucose level, aiming at 4.4-6.7 mmol/1. No fixed step-up regimen was available for HBGM or insulin therapy. In an interview after 1.5 years patients were asked if they had experienced hypoglycaemia.

The protocol was approved by the ethical committee of the Academic Hospital Vrije Universiteit.

Outcome measures. Fasting plasma glucose was determined with a glucose oxidase method (Boehringer Mannheim, Mannheim, Germany). Glycated haemoglobin $\left(\mathrm{HbA}_{1 \mathrm{c}}\right)$ was determined by ion-exchange high-performance liquid chromatography (HPLC), using a Modular Diabetes Monitoring System cose levels of $4.4-6.7 \mathrm{mmol} / \mathrm{l}$ and $\mathrm{HbA}_{1 \mathrm{c}}<7.0 \%$ (reference 4.3-6.1\%). ${ }^{\mathrm{b}}$ Neutral Protamin Hagendorn-insulin is an intermediate long-acting insulin

(Bio-Rad, Veenendaal, the Netherlands. normal range 4.3$6.1 \%$ ). In the control group, several methods of $\mathrm{HbA}_{1 \mathrm{c}}$ measurement were used (HPLC, affinity chromatography). $\mathrm{HbA}_{1 \mathrm{c}}$ values obtained with these methods were recalculated against those obtained with the HPLC-method by expressing these values as percentage of deviation of the upper limit of the normal range. Hypoglycaemia grade 3 was defined as that which required oral carbohydrates with help of others; a hypoglycaemic coma was scored as a hypoglycaemia grade 4 . Systolic and diastolic blood pressures were measured in the sitting position after $5 \mathrm{~min}$ rest with a digital blood pressure monitor (Omron Hem-405, Tokyo, Japan). Fasting total cholesterol, HDL-cholesterol and triglycerides were measured by 
Table 4. Change of glycaemic control, therapy and cardiovascular risk factors in patients known with NIDDM in study and control group

\begin{tabular}{|c|c|c|c|c|c|c|c|c|}
\hline & \multicolumn{3}{|c|}{ Study group $(n=350)$} & \multirow[t]{2}{*}{$P$-value } & \multicolumn{2}{|c|}{ Control group $(n=68)$} & \multirow[t]{2}{*}{$P$-value } & \multirow{2}{*}{$\begin{array}{l}\text { Study vs } \\
\text { control } \\
\text { group }\end{array}$} \\
\hline & baseline & year 1 & year 2 & & baseline & year 1.5 & & \\
\hline Fasting glucose $(\mathrm{mmol} / \mathrm{l})$ & $8.9 \pm 2.5$ & $8.4 \pm 2.6$ & $8.1 \pm 2.5$ & 0.000 & $9.6 \pm 3.4$ & $9.8 \pm 2.9$ & 0.74 & 0.004 \\
\hline BMI $\left(\mathrm{kg} / \mathrm{m}^{2}\right)$ & $28.7 \pm 4.6$ & $28.7 \pm 4.6$ & $29.0 \pm 4.6$ & 0.001 & $26.8 \pm 4.0$ & $26.5 \pm 3.8$ & 0.14 & 0.000 \\
\hline $\mathrm{HbA}_{1 \mathrm{c}}>8.5 \%(\%)$ & 21.4 & 12.1 & 11.7 & 0.000 & 23.5 & 27.9 & 0.58 & 0.008 \\
\hline $\mathrm{HbA}_{1 \mathrm{c}}<7.0 \%(\%)$ & 43.4 & 56.2 & 54.3 & 0.000 & 54.4 & 44.1 & 0.14 & 0.013 \\
\hline $\mathrm{SU} \pm$ metformin & 69.7 & 64.6 & 57.9 & 0.14 & 64.7 & 72.1 & 0.23 & \\
\hline SU + NPH-insulin at bedtime & 0.6 & 11.7 & 13.4 & 0.000 & 2.9 & 0 & 0.50 & \\
\hline Insulin bid & 2.9 & 8.3 & 16.3 & 0.000 & 2.9 & 8.8 & 0.13 & \\
\hline & \multicolumn{3}{|l|}{ c } & \multicolumn{2}{|l|}{ c } \\
\hline grade 3 (orally with help) & 0 & 0.006 & \multirow{2}{*}{$\begin{array}{l}0.006 \\
0.014\end{array}$} & \multicolumn{5}{|c|}{0} \\
\hline grade 4 (coma) & 0 & 0.011 & & & & 0 & & \\
\hline Diastolic blood pressure $(\mathrm{mm} \mathrm{Hg})$ & $87.4 \pm 10.8$ & $84.3 \pm 13.1$ & $\begin{array}{r}147.7 \pm 23.5 \\
830+126\end{array}$ & 0.000 & $88.8 \pm 11.4$ & $85.3 \pm 11.4$ & 0.01 & 0.59 \\
\hline Cigarette smokers (\%) & 22.0 & 24.0 & 18.0 & 0.003 & 23.9 & 20.9 & 0.50 & 0.75 \\
\hline
\end{tabular}

enzymatic techniques (Boehringer Mannheim, Mannheim, Germany). General well-being and treatment satisfaction were assessed with self-report questionnaires.

Statistical analysis. Baseline clinical characteristics were compared using chi-square, unpaired $t$ - or Mann-Whitney tests when appropriate. To compare baseline with final results paired $t$ - or $\mathrm{McNemar}$ tests were applied. For the comparison between study and control population corrected deltas ( = [baseline value - final value]/baseline value) were calculated and evaluated applying unpaired $t$-, Mann-Whitney-, and chi-square (Mantel-Haenszel method)-tests; the main endpoint (corrected delta $\mathrm{HbA}_{1 \mathrm{c}}$ ) was adjusted for differences in baseline characteristics applying linear regression analysis. To evaluate the change in general well-being and treatment satisfaction the Wilcoxon test was used. A p-value less than 0.05 was considered statistically significant.

\section{Results}

In the comparison between study and control population the 120 newly diagnosed NIDDM patients (with onset within 3 months) in the study group were omitted, since the control group did not include newly diagnosed patients. Baseline characteristics (Table 2) are comparable except for gender, fasting glucose, BMI, (HDL-) cholesterol and blood pressure.

Glycaemic control and blood glucose lowering therapy are depicted in Table 4 . In the study population a lowering of the $\mathrm{HbA}_{1 \mathrm{c}}$ was achieved within 1 year and then remained stable. The percentage of patients with poor glycaemic control fell from 21.4 to $\pm 12 \%$ and this result was maintained. Therefore, a comparison of the 2-year results in the study group with the 1.5-year results in the control group was allowed. The percentage of patients on insulin therapy increased gradually from 3.56 to $20 \%$ after 1 year to nearly $30 \%$ at 2 years. In these patients $(n=104)$ mean $\mathrm{HbA}_{1 \mathrm{c}}$ decreased from (mean \pm SD) $8.3 \pm 1.5$ to $7.6 \pm 1.3 \%$, with $2.4 \mathrm{~kg}$ mean weight gain. In patients not managed with insulin $(n=246)$, mean $\mathrm{HbA}_{1 \mathrm{c}}$ fell from $7.0 \pm 1.6$ to $6.8 \pm 1.2 \%$ and was associated with $0.4 \mathrm{~kg}$ mean weight loss. In newly diagnosed NIDDM patients followed for 2 years $(n=95$; Table 1$)$ mean $\mathrm{HbA}_{1 \mathrm{c}}$ decreased from 8.5 to $6.6 \%$ and the percentage with poor control from 43.2 to $9.5 \%$. Grade 3 hypoglycaemia was reported 4 times in 2 years, in patients treated with sulphonylures. On 13 occasions a hypoglycaemic coma was suspected. In 3 this was caused by a cardiac arrhythmia and in 1 by a transient ischaemic attack; glucose values during these episodes were all above $8.0 \mathrm{mmol} / \mathrm{l}$. The 9 remaining events occurred in 7 insulin-treated patients. These were unconfirmed and self-limiting (4) or corrected at home (5).

In the control group glycaemic control did not improve. The percentage of patients with poor glycaemic control, defined as $\mathrm{HbA}_{1 \mathrm{c}}$ above $8.5 \%$, tended to rise although insulin therapy was applied in $8.8 \%$. Exclusion of patients who needed insulin therapy (final $\mathrm{HbA}_{1 \mathrm{c}}$ values above $7.0 \%$ on maximal oral 
Table 5. Questionnaire for participating general practitioners of study group

\begin{tabular}{|c|c|c|}
\hline 1. Do you appreciate the co-operation with the diabetes service? & $\begin{array}{l}\text { yes } \\
\text { no }\end{array}$ & $\begin{array}{r}100 \% \\
0 \%\end{array}$ \\
\hline 2. Does participation in the shared care system save time? & $\begin{array}{l}\text { yes } \\
\text { equal } \\
\text { costs more }\end{array}$ & $\begin{array}{l}59 \% \\
24 \% \\
18 \%\end{array}$ \\
\hline 3. Without this shared care system, would you manage all these diabetic patients yourself? & $\begin{array}{l}\text { yes } \\
\text { no }\end{array}$ & $\begin{array}{l}17 \% \\
83 \%\end{array}$ \\
\hline $\begin{array}{l}\text { 4. Would you appreciate protocolized management of hypertension and lipid disorders with a } \\
\text { stepwise approach and concrete therapeutic advice? }\end{array}$ & $\begin{array}{l}\text { yes } \\
\text { no }\end{array}$ & $\begin{array}{l}76 \% \\
24 \%\end{array}$ \\
\hline 5. Do you enjoy the care for diabetic patients more since co-operation with the diabetes service? & $\begin{array}{l}\text { (very) much } \\
\text { a little } \\
\text { no change }\end{array}$ & $\begin{array}{r}72 \% \\
28 \% \\
0 \%\end{array}$ \\
\hline 6. Has glycaemic control in your patients been improved since shared care? & $\begin{array}{l}\text { (very) much } \\
\text { a little } \\
\text { no }\end{array}$ & $\begin{array}{r}72 \% \\
22 \% \\
6 \%\end{array}$ \\
\hline
\end{tabular}

therapy; $n=6$ control group), did not influence these results. Patients of the control group did not report hypoglycaemic episodes.

Mean diastolic blood pressure (both groups) and the number of cigarette smokers (study group) dropped significantly (Table 4). In the second year total cholesterol and triglyceride levels in patients of the study group decreased, partly due to the prescription of lipid modifying drugs. However, after 2 years of follow-up target values for blood pressure and lipid levels had still not been reached in most patients: hypertension ( $>160 / 95 \mathrm{mmHg}$ ) persisted in $30 \%$; hypercholesterolaemia $(>6.5 \mathrm{mmol} / \mathrm{l})$ in $25 \%$ and hypertriglyceridaemia $(>2.2 \mathrm{mmol} / \mathrm{l})$ in $29 \%$. When applying more stringent criteria for hypertension (>140/90 $\mathrm{mmHg}$ ), total cholesterol $(>5.2 \mathrm{mmol} / \mathrm{l})$ and triglycerides $(>1.7 \mathrm{mmol} / \mathrm{l})$, these percentages were 56,70 and $52 \%$, respectively.

General well-being and treatment satisfaction were evaluated only in the study group. Of 247 of the 445 patients $(55.5 \%)$ a questionnaire at baseline and after 2 years was available. These patients did not differ from those who did not return the questionnaire for age, gender, diabetes duration, BMI and improvement of $\mathrm{HbA}_{1 \mathrm{c}}$. General well-being ('how have you felt in the past 3 months' and 'how do you judge your health') did not show a significant change ( $p$-value 0.36 and 0.49 , respectively). Questions concerning medical care in the past 3 months and whether improvement of medical care was possible in the opinion of the patient tended to improve ( $p$-value 0.46 and 0.15 , respectively). The patients tended to find the diabetes management less demanding during the study than before ( $p$-value $0.06)$. GPs all appreciated the co-operation with the diabetes service (Table 5). The diabetes nurse played an important role in education and adjustment of the insulin dose; only 4 out of 22 GPs personally adjusted the insulin doses.

\section{Discussion}

A lasting improvement in blood glucose control in a large population of NIDDM patients was achieved in primary health care as a result of implementing a structured diabetes programme. Good control, defined as $\mathrm{HbA}_{1 \mathrm{c}}$ less than $7.0 \%$, was achieved in the majority of patients. The percentage of patients with poor control $\left(\mathrm{HbA}_{1 \mathrm{c}}>8.5 \%\right)$ was nearly halved to $\pm 12 \%$. In the control population it proved difficult to implement protocolized diabetes care according to the GP guidelines. More than a quarter of the patients in the control group remained poorly regulated.

Differences in baseline characteristics in the study and control populations do not explain the observed differences in glycaemic control. Neither do treatment targets or guidelines. Our data strongly suggest that the difference cannot be attributed to the availability of insulin therapy; there are two more likely explanations for the better results in the study group. Firstly, the protocolized therapy advice, based upon the results and pre-set targets, were in general followed by the GPs; they were requested to report their reasons for not doing so. GPs reported increased enthusiasm after 2 years of participation, probably reflecting a positive attitude towards diabetes care. Secondly, individual diabetes education (e.g. by diabetes nurse and dietitian) may have enhanced self-care and patient compliance [15]. Patient motivation is also reflected by the fact that, by using blood glucose selfmonitoring, they were easily motivated to start insulin therapy. Of the 579 patients in the study group only $20(3.5 \%)$ dropped out because of poor motivation. The main reasons for this were psychiatric problems, alcohol addiction and short life expectancy due to cancer.

Nearly $30 \%$ of the patients in the study group were treated with insulin at 2 years of follow-up. The combination of insulin at bedtime with sulphonylurea drugs, as advocated in NIDDM [24-26], followed by 
NPH-insulin twice daily, appeared to be an effective, feasible and safe therapy regimen. The incidence rate of severe hypoglycaemia was low, 0.019 /patient year for the whole group and 0.064 /insulin treated patient year. This low frequency is in agreement with the findings of the VA CSDM feasibility trial $(0.03$ / patient year intensive therapy group) [27] and the UKPDS (0.014/insulin treated patient year) [28]. The incidence rate in IDDM is much higher (DCCT: 0.74 /patient year) [29]. In comatose patients it appeared to be difficult to distinguish hypoglycaemia from cardiac arrhythmia or transient ischaemic attack as was the case in 4 of the 13 events.

In contrast to our favourable findings for glycaemic control, a considerable number of patients did not achieve targets for serum lipid levels, blood pressure and smoking [2,4]. Although the study group performed slightly better than the control group, more vigorous management programmes are needed to reduce the number of cardiovascular risk factors. As with the management of hyperglycaemia, a protocolized stepwise treatment scheme based upon accepted guidelines for the management of dyslipidaemia and hypertension may be the method of choice.

One of the remarkable findings was that general well-being did not worsen under the strict metabolic control, although insulin therapy was initiated in nearly $30 \%$ of patients. Also, patients were satisfied with the medical care they experienced during follow-up. It is unlikely that improved glycaemic control is the only explanation for these positive answers. It is more likely that the patients appreciated the easily available diabetes control, education and consultation facilities.

The 22 GPs in the study group all responded favourably to the questions referring to co-operation with the diabetes service at the GP laboratory. In particular the assistance of the diabetes nurse educator and the consultation facilities with the diabetologist were felt as important support when transferring patients to insulin therapy.

This study clearly shows that implementation of structured care in general practice results in sustained good glycaemic control in the majority of NIDDM patients. Insulin therapy could safely be applied in primary care. In contrast, just providing laboratory results without defined therapeutic advice only resulted in a modest lowering of cardiovascular risk factors. Finally, this shared care system was well appreciated, both by patients and professionals.

Acknowledgements. We thank Elise Kuipers, dietitian, Lucie Ernst and Joke Kortekaas, diabetes nurse educators, and the participating GPs and their assistants for all the hard work they put into this shared care system. We are obliged to Piet van der Wal for designing the computer programme. Frank Snoek, medical psychologist, is thanked for his advice concerning evaluation of well-being. We thank Erica de Ruyter and
Arno van der Weert, medical students, for screening of all GP patient records.

This study was supported by the Research Centre Primary/Secondary Health Care at the Academic Hospital Vrije Universiteit. Hoechst Marion Roussel Nederland BV and Medisense Nederland BV provided additional research grants.

\section{References}

1. World Health Organization (Europe) and International Diabetes Federation (Europe) (1990) Diabetes care and research in Europe: The St. Vincent declaration. Diabet Med 7: 360

2. Alberti KGMM, Gries FA (1988) Management of non-insulin-dependent diabetes in Europe: a consensus view. Diabet Med 5: 275-281

3. Alberti KGMM, Gries FA, Jervell J. Krans HMJ for the European NIDDM Policy Group (1994) A desktop guide for the management of non-insulin-dependent diabetes mellitus (NIDDM). An Update. Diabet Med 11: 889-909

4. Cromme PVM, Mulder JD, Rutten GEHM, Zuidweg J. Thomas S (1993) NHG-standard diabetes mellitus type II. In: Rutten GEHM, Thomas S (eds) Guidelines for the general practitioner. [In Dutch: NHG-standaarden voor de huisarts.] Bunge, Utrecht, pp 132-140

5. Grol R (1990) National standard setting for quality of care in general practice: attitudes of general practitioners and response to a set of standards. Br J Gen Pract 40: 23-25

6. Chesover D, Tudor-Miles P, Hilton S (1991) Survey and audit of diabetes care in general practice in south London. $\mathrm{Br}$ J Gen Pract 41: 282-285

7. Jacques CHM, Jones RL, Houts P et al. (1991) Reported practice behaviors for medical care of patients with diabetes mellitus by primary-care physicians in Pennsylvania. Diabetes Care 14: 712-717

8. Higgs ER, Kelleher A, Simpson HCR, Reckless JPD (1992) Screening programme for microvascular complications and hypertension in a community diabetic population. Diabet Med 9: 550-556

9. Pringle M, Stewart-Evans C, Coupland C, Williams I, Allison S, Sterland J (1993) Influences on control in diabetes mellitus: patient, doctor, practice or delivery of care. BMJ 306: 630-634

10. Benett IJ, Lambert C, Hinds G, Kirton C (1994) Emerging standards for diabetes care from a city-wide primary care audit. Diabet Med 11: 489-492

11. Querido JD, Von Veh V (1995) Caring for type-2 diabetics in three inner city practices. Assessment of a protocol. Huisarts Wet 38(6):246-249

12. Whitford D, Avery A (1989) Barriers to comprehensive diabetes care in the northern region. Practical Diabetes 6: 114-116

13. Jones JN, Marsden P (1992) Improved diabetes care in a UK health district. Diabet Med 9: 176-180

14. Roland G, Hiss MD (1996) Barriers to care in non-insulindependent diabetes mellitus. The Michigan experience. Ann Intern Med 124: 146-148

15. Assal JP, Mühlhauser I, Pernet A, Gfeller R, Jörgens V, Berger M (1985) Patient education as the basis for diabetes care in clinical practice and research. Diabetologia 28: 602613

16. Singh BM, Holland MR, Thorn PA (1984) Metabolic control of diabetes in general practice clinics: comparison with a hospital clinic. BMJ 289: 726-728 
17. Koperski M (1992) How effective is systematic care of diabetic patients? A study in one general practice. Br J Gen Pract 42: 508-511

18. Thorell B, Olsson L, Svardsudd K (1994) Implementation of a structured care programme for diabetes mellitus in a defined population in mid-Sweden. Diabet Med 11: 458-464

19. Diabetes Integrated Care Team (1994) Integrated care for diabetes: clinical, psychological, and economic evaluation. BMJ 308:1208-1212

20. Jacobs ML, Akkerhuis KM, Dijk MJ van, Kuis FBM, Veldkamp RTM, Weber RFA (1995) Better diabetes control by strict observance of the standard 'Diabetes mellitus type II' of the Dutch College of General Practitioners. Ned Tijdschr Geneesk 139: 1241-1245

21. Diabetes and Nutrition Study Group of the European Association for the Study of Diabetes (1995) Recommendations for the nutritional management of patients with diabetes mellitus. Diab Nutr Metab 8: 1-4

22. Sonnaville JJJ de, Feltz van der Sloot D van der, Ernst L, Wijkel D, Heine RJ (1996) Retinopathy screening in type II diabetes. Reliability of wide angle fundus photography. Diabet Med 13: 482-486

23. Sonnaville JJJ de, Wijkel D, Heine RJ (1997) The prevalence and determinants of foot ulceration in type II diabetic patients in a primary health care setting. Diab Res Clin Pract 35: 149-156
24. Yki-Järvinen H, Kauppila M, Kujansuu E et al. (1992) Comparison of insulin regimens in patients with non-insulin-dependent diabetes mellitus. N Engl J Med 327: 14261433

25. Chow CC, Sorensen JP, Tsang LWW, Cockram CS (1995) Comparison of insulin with or without continuation of oral hypoglycaemic agents in the treatment of secondary failure in NIDDM patients. Diabetes Care 18: 307-314

26. Johnson JL, Wolf SL, Kabadi UM (1996) Efficacy of insulin and sulfonylurea combination therapy in type II diabetes. Arch Intern Med 156: 259-264

27. Abraira C, Colwell JA, Nuttall FQ et al. (VA CSDM Group) (1995) Veterans Affairs Cooperative Study on Glycemic Control and Complications in type II diabetes (VA CSDM). Diabetes Care 18: 1113-1123

28. Holman RR, Cull CA, Fox C, Turner RC, United Kingdom Prospective Study Group (1995) United Kingdom prospective diabetes study (UKPDS) 13: relative efficacy of randomly allocated diet, sulphonylurea, insulin, or metformin in patients with newly diagnosed non-insulin dependent diabetes followed for three years. BMJ 310: 83-88

29. The Diabetes Control and Complications Trial Research Group (1993) The effect of intensive treatment of diabetes on development and progression of long term complications in insulin dependent diabetes mellitus. N Eng J Med 329: $977-986$ 